\title{
Construction of Plasmids Expressing Sars-CoV Encoding Proteins and Their Effects on Transcription of Hfgl2 Prothrombinase*
}

\author{
Hongwu WANG (王洪武 $)^{1 \dagger}$, Meifang HAN (韩梅芳 $)^{1 \dagger}$, Huaning YAO (姚华宁 $)^{1}$, Zhanhui WANG (王战会 $)^{2}$, Dong XI (习东 $)^{1}$, \\ Weiming YAN (严伟明) $)^{1}$, Jinlin HOU (侯金林 $)^{2}$, Xiaoping LUO (罗小平 $)^{3}$, Qin NING (宁 琴) $)^{1 \#}$ \\ ${ }^{I}$ Department of Infectious Disease, ${ }^{3}$ Department of Pediatrics, Tongji Hospital, Tongji Medical College, Huazhong University of \\ Science and Technology, Wuhan 430030, China \\ ${ }^{2}$ Department of Infectious Diseases, Nanfang Hospital, Nanfang Medical University, Guangzhou 510515, China
}

\begin{abstract}
Summary: SARS coronavirus (SARS-CoV) is the etiologic agent of severe acute respiratory syndrome. The aim of this study was to construct Sars-CoV membrane (M), nucleocapsid (N) and spike 2 (S2) gene eukaryotic expression plasmids, and identify their expression in vitro. Gene fragments encoding $\mathrm{N}$ protein, $\mathrm{M}$ protein and $\mathrm{S} 2$ protein of SARS-CoV were amplified by PCR using cDNA obtained from lung samples of SARS patients as template, and subcloned into pcDNA3.1 vector to form eukaryotic expression plasmids. SARS-CoV protein eukaryotic expression plasmids were transfected respectively into CHO cells. Immunohistochemistry was employed to detect the expression of the structural proteins of SARS-CoV in transfected cells. SARS-CoV protein eukaryotic expression plasmids were successfully constructed by identification with digestion of restriction enzymes and sequencing. $\mathrm{M}, \mathrm{N}$ and $\mathrm{S} 2$ proteins of SARS-CoV were detected in the cytoplasm of transfected $\mathrm{CHO}$ cells. It was concluded that these recombinant eukaryotic expression plasmids were constructed successfully, and SARS-CoV encoding proteins could activate transcription and expression of hfgl2 gene.
\end{abstract}

Key words: SARS-CoV; encoding protein; gene expression

SARS-associated coronavirus (SARS-CoV) is the etiologic agent of severe acute respiratory syndrome (SARS), which spread worldwide in 2003, infecting thousands of people and killing hundreds ${ }^{[1]}$. The high viral virulence resulting in a significant mortality rate of infected patients has created widespread scientific interest in understanding the mechanisms of pathogenicity of this virus. Molecular biological analyses of SARS-CoV identified 14 open reading frames (ORFs) ${ }^{[2]}$. The SARS$\mathrm{CoV}$ genome encodes four structural proteins: spike $(\mathrm{S})$, envelope (E), membrane (M) protein and nucleocapsid protein $(\mathrm{N})^{[2-4]}$. These proteins play a role in viral replication and packaging, as well as the process of invasion, respectively ${ }^{[5,6]}$. Studies have shown that these viral proteins can serve as not only a structural protein involved in the synthesis of the virus, but also a regulatory protein in the regulation of host gene expression ${ }^{[7-10]}$.

Fibrinogen-like protein-2 (Fgl2) prothrombinase is also named fgl2 fibroleukin, and fgl2 plays an important role in the development of murine hepatitis virus type 3 (MHV-3)-induced fulminant hepatitis ${ }^{[11-14]}$, human se-

\footnotetext{
Hongwu WANG, E-mail: hongwuwang@126.com

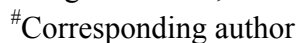

${ }^{\dagger}$ Hongwu WANG and Meifang HAN contributed equally to the work.

*This project was supported by a grant from National Key Project of Science and Technology Ministry of China for 973-SARS (No. 2003CB514112), SARS funding first granted from Ministry of education of China ([2003]64), and The National 10th Five-Year Plan Key Project of China (2004BA720A01).
}

vere acute chronic hepatitis $\mathrm{B}^{[15,16]}$, experimental and human allograft rejection and cancer ${ }^{[17]}$.

SARS-CoV can cause inflammation of lung tissue associated with edema, hemorrhage and necrosis, cellulose deposition, accompanied by a wide range of coagulation dysfunction. In preliminary studies we have confirmed fgl2 gene expression in murine SARS model ${ }^{[11-13]}$. Foreign scholars have also noticed that hfgl2 genes may be involved in the formation of immune-coagulation in SARS patients ${ }^{[17-21]}$. Whether the SARS-CoV protein coding genes contribute to hfgl2 activation thus promoting the occurrence and development of hemorrhagic necrosis, fibrin deposition and microcirculatory disturbance in lung tissues, is still unclear. In this study, we established the eukaryotic expression vectors of SARS-CoV encoding proteins S2, M and E, and co-tranfected them into $\mathrm{CHO}$ cells with the hfgl2 promoter luciferase-reporter gene to investigate the activation effect of SARS$\mathrm{CoV}$ encoding proteins on hfgl2 gene.

\section{MATERIALS AND METHODS}

\subsection{Construction of Eukaryotic Expression Plasmids pcDNA-N, pcDNA-M and pcDNA-S2}

The construction of prokaryotic expression vectors pET28-S2, pET28-N and pET32-M were described previously ${ }^{[20]}$. Briefly, total RNA was extracted from the lung tissues of SARS patients confirmed by the diagnostic standard of Ministry of Public Health announced on April 5th, 2003. RT-PCR was performed according to the manufacturer's instructions. S2, M and N genes were amplified by PCR using the cDNA as template. S2 and N genes of SARS were cloned into the prokaryotic expression vector pET28, while $M$ gene was cloned into the 
vector pET32. SARS-CoV S2 and $M$ gene fragments were released from pET28-S2 and pET32-M by digestion with $B a m \mathrm{H}$ I and Xho I, and then subcloned into eukaryotic expression vector pcDNA3.1 (+) using the $B a m \mathrm{H}$ I and Xho I sites to form pcDNA-S2 and pcDNA-M. SARS-CoV N gene fragments were released from pET28-N by digestion with BamH I and Not I, and then subcloned into pcDNA3.1 (+) using the BamH I and Not I sites to form eukaryotic expression plasmid pcDNA-N.

\subsection{Construction of Hfgl2 Promoter Luciferase-re- porter Plasmids}

Fg12 promoter luciferase-reporter plasmids were constructed as described previously ${ }^{[14]}$. Briefly, a $1.3-\mathrm{kb}$ DNA fragment including the entire hfgl2 gene promoter was released by restriction digestion with EcoRV and Sal I from a subclone pBluescript-m166 (pm166) of human genomic P1 plasmid (Genome System Inc, USA) that contained the entire hfgl2 gene. The isolated $1.3-\mathrm{kb}$ fragment was then inserted into SmaI and XhoI sites of the pGL2-Basic luciferase-reporter vector (pGL2-Basic, Promega, USA) to form hfgl2 promoter luciferase-reporter plasmid hfgl2p(-1334)LUC ${ }^{[14]}$. 5'-truncation of the hfgl2 promoter in pGL2-Basic was carried out by PCR using hfgl2p(-1334)LUC as template. The series PCR products were respectively cloned into PCR2.1 cloning vector, and subcloned into pGL2-Basic plasmid at Hind III and XhoI sites to form four 5'-truncation of hfgl2 promoter luciferase-reporter constructs. The upstream primers used were 5'-CTT ATG TCT TTC CTG CCT TC- 3' for hfgl2p(-998)LUC, 5'-GGC AAG AGA AGT TCA GGA C-3' for hfgl2p(-817)LUC, 5'-AAT ACA GGC TCC CCA ATG C- 3' for hfgl2p(-467)LUC and 5' -GTG AAT CTT GTT GGC TGT G-3' for hfgl2p(-243)LUC. The common downstream primer used was 5'-TTC GCC CAT CTT TAC AGTG-3', respectively. The promoter luciferase-reporter plasmids were all sequenced to confirm the orientation and verify the sequence.

\subsection{Cell Culture and Transient Transfections}

CHO cell line was cultured with F12 medium (Gibco, USA) and human macrophage cell line THP-1 with 1640 medium, and both media were supplemented with $10 \%$ FBS. Cells were plated in 6-well culture plates at $50 \%$ to $70 \%$ confluence. Two $\mu \mathrm{g}$ of expression plasmid pcDNA-N, pcDNA-M or pcDNA-S2, $1 \mu \mathrm{g}$ hfgl2p(-1334)LUC plasmid, and $0.5 \mu \mathrm{g} \beta$-galactosidase plasmid (as a marker for transfection efficiency by $\beta$-galactosidase assay; Rous sarcoma virus $\beta$-galactosidase vector was purchased from Promega, USA) in $100 \mu \mathrm{L}$ Opti-MEM medium (Invitrogen, USA) were mixed by vortexing with $8 \mu \mathrm{L}$ of Lipofectamine 2000 (Invitrogen, USA) in $100 \mu \mathrm{L}$ Opti-MEM medium. After incubation of the mixture at room temperature for $20 \mathrm{~min}, 1.8 \mathrm{~mL}$ Opti-MEM medium (Invitrogen, USA) was added to bring up the volume to 2 $\mathrm{mL}$. One $\mathrm{mL}$ of the mixture was distributed into one of the duplicated wells with $\mathrm{CHO}$ cells or THP-1 cells. After cells were cultured for $10-12 \mathrm{~h}$, another $1 \mathrm{~mL}$ culture medium was added to the well. Transfection was performed at $37^{\circ} \mathrm{C}$ with $5 \% \mathrm{CO}_{2}$ for $40-44 \mathrm{~h}$. After transfection, these cells were harvested for detection of the expression of SARS-CoV M, N and S2 proteins by immunocytochemistry.

\subsection{Immunocytochemistry}

Cells transfected with pcDNA-M, pcDNA-N or pcDNA-S2 were fixed with $80 \%$ acetone for $10 \mathrm{~min}$, and then incubated in $3 \% \mathrm{H}_{2} \mathrm{O}_{2}$ to block internal peroxidase for $10 \mathrm{~min}$ at room temperature. Then cells were incubated for $10 \mathrm{~min}$ at room temperature in blocking solution of normal goat serum according to the manufacturer's instructions (SP kits, Sigma, USA). The cells were stained with the anti-SARS-CoV-M, anti-SARSCoV-N or anti-SARS-CoV-S2 polyclonal antibodies from rabbit (provided by Prof. Hu Zhihong, Key Laboratory of Molecular Virology, Wuhan Institute of Virology, Chinese Academy of Sciences Wuhan Virus Research, China) with a dilution of 1:200 overnight followed by a secondary biotinylated anti-rabbit IgG at a dilution of 1:1000. Solution of streptavidin-peroxidase was added to cells. As negative controls, CHO cells, either without transfection or transfected with pcDNA3.1(+) empty vector were employed.

\subsection{Real-time Fluorescence Quantitative RT-PCR}

Previous study found that the hfgl 2 expression is confined to macrophage cells, lymphocytes, and endothelial cells of intrahepatic veins and hepatic sinusoids. Therefore THP-1 cell line system was introduced to characterize hfgl2 gene transcript and protein expression in response to SARS-CoV proteins. Total RNA was extracted from THP-1 cells transfected with pcDNA-M, pcDNA-N or pcDNA-S2 with TRIzol reagent (Invitrogen, USA) according to the manufacturer's standard protocol. Real-time fluorescence quantitative RT-PCR was done with EvaGreen PCR reagents (Biotium, USA) according to the manufacturer's standard protocol to detect the mRNA level of hfgl2. The upstream and down primers were 5'-ACT GTG ACA TGG AGA CCA TG-3', and 5'-TCC TTA CTC TTG GTC AGA AG-3' respectively.

\subsection{Western Blot Analysis}

The supernatant of THP-1 cells lysate transfected with pcDNA-M, pcDNA-N or pcDNA-S2 was detected for the expression of hfgl2 protein using polyclonal antibody from rabbit against hfgl2 prothrombinase (1:500 dilution). After washing 5 times, the membranes were probed with a horseradish peroxidase-labeled goat anti-rabbit secondary antibody (Santa Cruz Biotechnology, USA) for $1 \mathrm{~h}$. The peroxidase based detection was performed with Super Signal West Pico Chemiluminescent Substrates Kits (Pierce, USA) according to the manufacturer's instructions.

\subsection{Luciferase Assay and Mapping of the Hfgl2 Pro- moter}

Cells transfected with pcDNA-N, pcDNA-M, or pcDNA-S2, luciferase-reporter vector $h f g 12 p(-1334)$ LUC were collected and lysed in buffer containing 150 $\mathrm{mmol} / \mathrm{L} \mathrm{NaCl}, 50 \mathrm{mmol} / \mathrm{L}$ Tris, $5 \mathrm{mmol} / \mathrm{L}$ EDTA, $1 \%$ Nonidet P-40, and protease inhibitor for $30 \mathrm{~min}$ on ice, and then the supernatant of cell lysate was obtained by subsequent centrifugation for Western blotting. Luciferase activity and $\beta$-galactosidase activity were assayed by using the luciferase and $\beta$-galactosidase enzyme assay system (Promega, USA). Luciferase activity was normalized with the $\beta$-galactosidase activity in cell lysate, and calculated as an average of three independent experiments. PGL2-Basic vector was used as a negative 
control. A series of $5^{\prime}$ truncated hfgl2 promoter/report constructs hfgl2p(-1334)LUC, hfgl2p(-998)LUC, hfgl2p(-817)LUC, hfgl2p(-467)LUC and hfgl2p(-243) LUC was respectively co-transfected with pcDNA-N and $\beta$-galactosidase plasmid to $\mathrm{CHO}$ cells as previously described. After incubation for 40-44 h, cells were lysed to detect the luciferase activity and $\beta$-galactosidase enzyme activity.

\subsection{Statistical Analysis}

Data were expressed as $\bar{x} \pm s$ where applicable. Student's $t$ test for unpaired samples (two-tailed) was used to analyze the data using the SPSS 12.0 statistical software.

\section{RESULTS}

2.1 Identification of pcDNA-N, pcDNA-M and pcDNA-S2

Electrophoretic identification of recombinant plasmids pcDNA-N, pcDNA-M and pcDNA-S2 by restriction enzyme was done (fig. 1). Agarose gel electrophoresis showed that pcDNA-M and pcDNA-S2 could be digested by BamH I and Xho I restriction enzyme with 666 and $1653 \mathrm{bp}$ fragments respectively after $3 \mathrm{~h}$ digestion, and pcDNA-N by BamH I and Not I restriction enzyme with a 1266 bp fragment after digestion. The orientation and gene sequences of these three plasmids were confirmed by DNA sequencing.

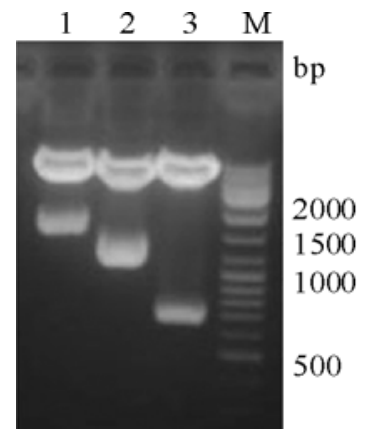

Fig. 1 Identification of recombinant pcDNA-S2, pcDNA-N and pcDNA-M plasmids by enzyme digestion 1: pcDNA-S2/BamH I and Xho I; 2: pcDNA-N/BamH I and Not I; 3: pcDNA-M/BamH I and Xho I; M: DNA marker

\subsection{Identification of Hfgl2 Promoter Luciferase-re- porter Plasmids}

Electrophoretic identification of recombinant plasmids hfgl2p(-998)LUC, hfgl2p(-817)LUC, hfgl2p(-467) LUC and hfglp2(-243)LUC by restriction enzyme was done (fig. 2). Agarose gel electrophoresis showed that hfgl2p(-998)LUC, hfgl2p(-817)LUC, hfgl2p(-467)LUC and hfglp2(-243)LUC could be digested by Hind III and Xho I restriction enzyme with 1008, 827, 477 and 253 bp fragments respectively after digestion. All above sequences were confirmed by DNA sequencing.

\subsection{Expression of SARS-CoV N, M and S2 Proteins in CHO Cells}

To detect the expression of SARS-CoV N, M and $\mathrm{S} 2$ proteins in $\mathrm{CHO}$ cells, immunocytochemistry was used to $\mathrm{CHO}$ cells transfected with eukaryotic expression vectors pcDNA-N, pcDNA-M or pcDNA-S2. The dark brown cytoplasmic staining was seen in either pcDNA-N, pcDNA-M or pcDNA-S2 transfected cells by immunocytochemistry (fig. 3), but there was no or trace amount of staining in pcDNA3.1 empty vector transfected cells (negative control).

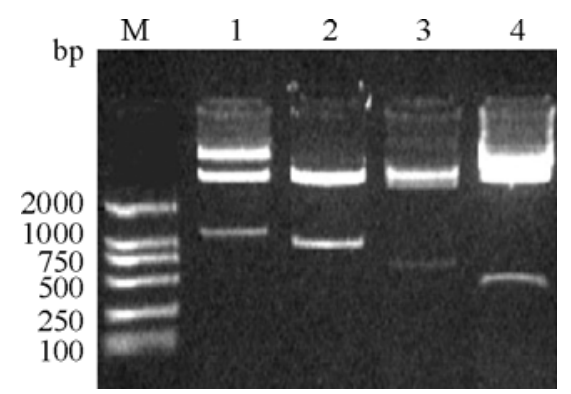

Fig. 2 Identification of progressive deletions of hfgl2 promoter luciferase-reporter constructions by enzyme digestion

1: hfgl2p(-998)LUC; 2: hfgl2p(-817)LUC;

3: hfgl2p(-467) LUC; 4: hfgl2p(-243)LUC. All the recombinants were digested with Hind III and Xho I restriction enzymes

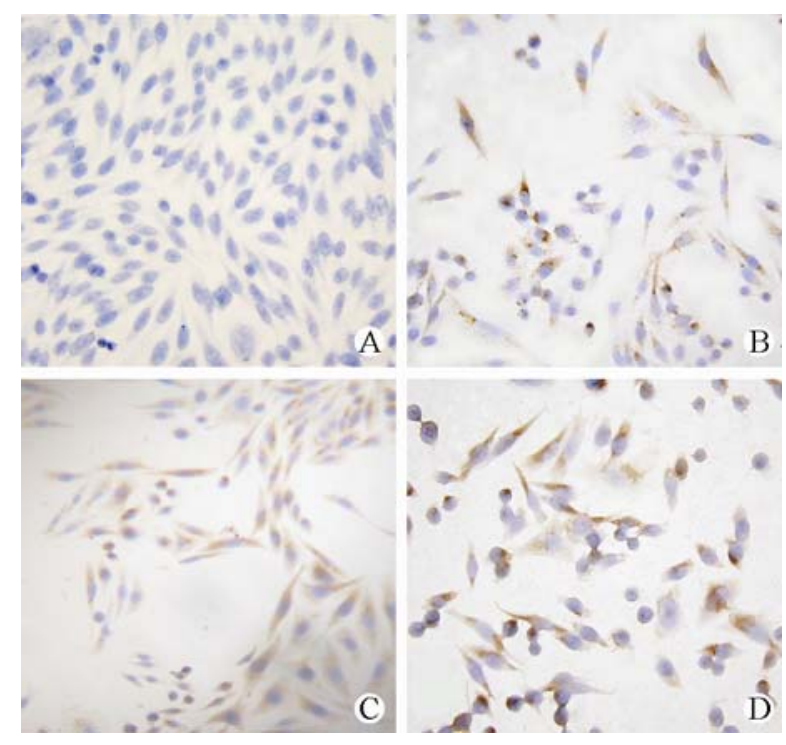

Fig. 3 Expression of SARS-CoV N, M and S2 proteins in $\mathrm{CHO}$ cells $(\mathrm{SP} \times 200)$

A: $\mathrm{CHO}$ cells transfected with pcDNA3.1(+), as negative control;

B: CHO cells transfected with pcDNA3.1-N;

C: $\mathrm{CHO}$ cells transfected with pcDNA3.1-M;

D: CHO cells transfected with pcDNA3.1-S2

\subsection{Effect of SARS-CoV N, M and S2 Proteins on Hfgl2 Expression}

To evaluate the effects of SARS-CoV encoding proteins on hfgl 2 expression in vitro, a time course study of the effects was performed by real-time fluorescence quantitative RT-PCR, using THP-1 cells transfected with pcDNA-N, pcDNA-M or pcDNA-S2 for 40-44 h (fig. 4). It was evident that SARS-CoV $\mathrm{N}$ protein significantly enhanced hfgl2 mRNA expression as compared with $\mathrm{M}$ and S2 proteins. To detect the expression level of hfgl2 
protein in response to SARS-CoV proteins, Western blot analysis of hfgl 2 was performed. It was found that hfgl2 protein was enhanced distinctly by the stimulation of SARS-CoV N protein. No hfgl2 protein was detected in the cells transfected with pcDNA-M or pcDNA-S2 (fig. $5)$.

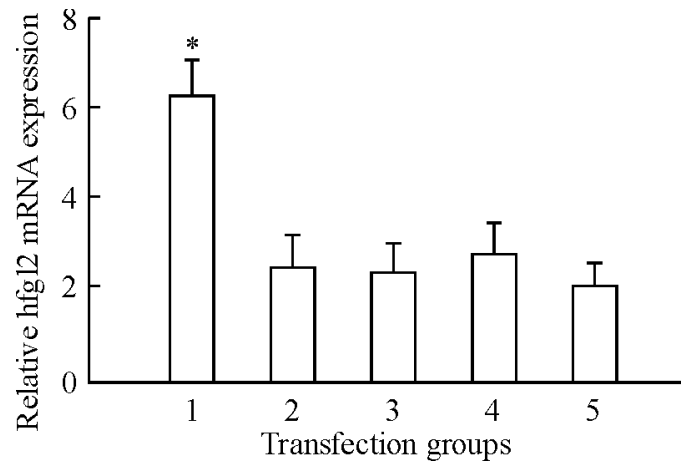

Fig. 4 Real-time PCR analysis of the expression of hfgl2 in response to SARS-CoV N, M and S2 proteins THP-1 cells were transfected with pcDNA-N (1), pcDNA-M (2), pcDNA-S2 (3) and pcDNA-3.1 empty vector (4) respectively, and untransfected cells (5) as control group. Values are expressed as $\bar{x} \pm s$ of three separate experiments done in triplicate. ${ }^{*} P<0.01$ compared with control group

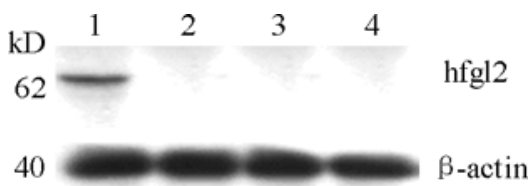

Fig. 5 Western blot analysis of the hfgl2 expression in response to SARS-CoV N, M and S2 proteins. THP-1 cells were transfected with pcDNA-N (1), pcDNA-M (2), pcDNA-S2 (3) and pcDNA-3.1 empty vector (4) respectively

\subsection{Mechanism of SARS-CoV N Protein-induced Hfgl2 Transcription}

To explore the mechanism of increased mRNA and protein levels of hfgl2 in response to SARS-CoV proteins, CHO cells were co-transfected with the hfgl2 promoter construct hfgl2p(-1334)LUC and pcDNA-N, pcDNA-M or pcDNA-S2 vectors. As shown in fig. 6, consistent with real-time PCR and Western blot results, SARS-CoV N protein induced hfgl 2 promoter activity with an average increasing of 6.0 -fold in $\mathrm{CHO}$ cells as compared with that in cells co-transfected with pcDNA3.1 empty vector (fig. 6). There was no significant change in relative luciferase activity when pcDNA-M or pcDNA-S2 was co-transfected with hfgl2p(-1334)LUC in CHO cells. These results suggested that SARS-CoV N protein but not $\mathrm{M}$ or S2 protein induces hfgl2 promoter activity in $\mathrm{CHO}$ cells.

\subsection{Mapping of the Hfgl2 Promoter}

To characterize the region in the hfgl 2 promoter which is responsive to $\mathrm{N}$ protein of SARS-CoV, constructs containing progressive deletion of the hfgl2 promoter was co-transfected with pcDNA-N and a $\beta$-galactosidase vector in $\mathrm{CHO}$ cells. As shown in fig. 7, preliminary mapping of the hfgl2 promoter has defined a region from -817 to -467 to be responsive to induction of $\mathrm{N}$ protein of SARS-CoV. Using bioinformatics software (TESS and TFSEARCH, Computational Biology Research Center, AIST, Japan), some putative cis-acting regulatory transcription factors were identified within this region, such as a tinman homeodomain factor (Nkx-2), lymphocyte enhancer factor-1 (LEF-1), c-Ets-2 (v-ets erythroblastosis virus E26 oncogene homolog 2), heat shock factor (HSTF) and so on.

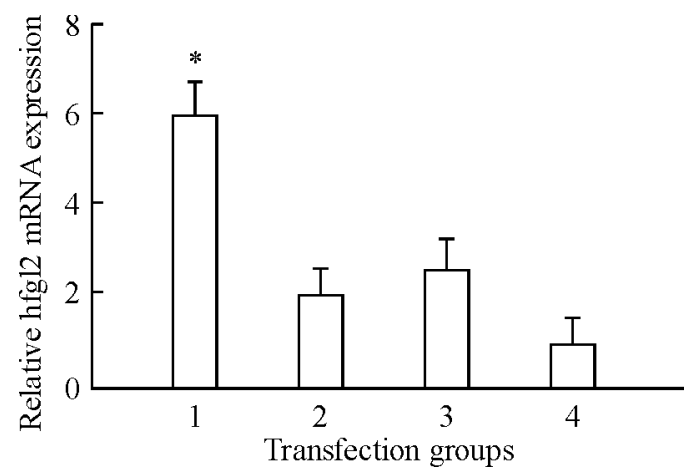

Fig. 6 Effects of SARS-CoV proteins on transcriptional activation of hfgl2 in CHO cells. pcDNA-N (1), pcDNA-M (2) or pcDNA-S2 (3) was co-transfected with hfgl2p (-1334)LUC in CHO cells for $40-44 \mathrm{~h}$, and the cells were harvested for measurement of luciferase activity. Values are expressed as $\bar{x}_{ \pm s}$ of four separate experiments. ${ }^{*} P<0.01$ as compared with cells co-transfected with empty pcDNA3.1 vector (4)

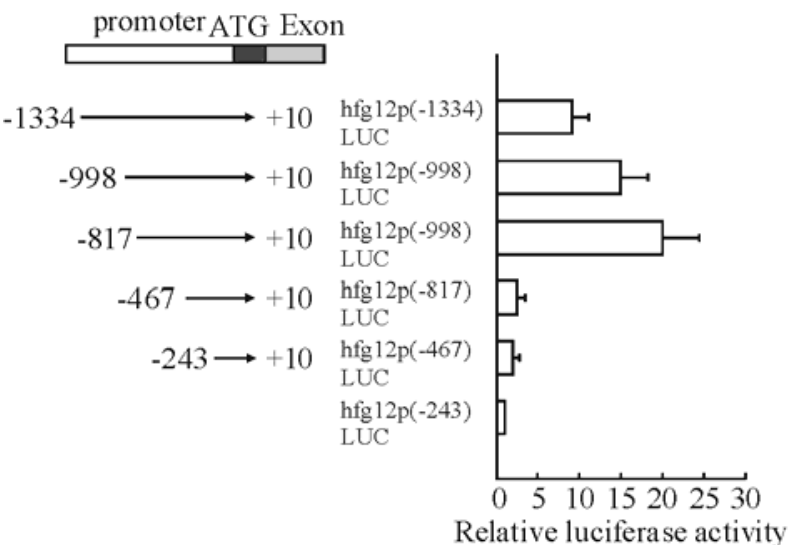

Fig. 7 Effects of SARS-CoV nucleocapsid protein on transcriptional activation of a series of hfgl2 promoter in $\mathrm{CHO}$ cells. pcDNA-N was co-transfected with progressive deletions of hfgl 2 promoter luciferase-reporter constructions into $\mathrm{CHO}$ cells. The cells transfected with pGL2-Basic vectors served as control group. After transfection for $40-44 \mathrm{~h}$, cells were harvested for measurement of relative luciferase activity

\section{DISCUSSION}

Since severe acute respiratory syndrome broke out in November 2002, of more than 8422 patients who contracted this highly infectious disease, 908 have died. 
SARS-CoV has been confirmed as the pathogen. Post-mortem lung samples from 6 patients who died of SARS from April to July 2003 were studied. Morphologic changes are summarized as diffuse and bilateral lung consolidation and diffuse alveolar damage with hyaline membrane formation. Intra-alveolar edema/ hemorrhage, fibrin deposition, pneumocyte desquamation was universal ${ }^{[21]}$. Diffuse alveolar damage and fibrin deposition in SARS prompt the study that the hfgl2 gene is expressed highly in SARS substitutive animal model ${ }^{22]}$. Through this study we constructed three eukaryotic expression constructions of SARS-CoV proteins successfully and first confirmed that SARS-CoV N protein activated the expression of hfgl2 gene in vitro, which may partially explain the fibrin deposition and diffuse alveolar damage in SARS patients.

The results in this study demonstrated that the nucleocapsid protein of SARS induced transcription of hfgl2. Fgl2 prothrombinase has been cloned and identified to belong to fibrinogen proteins family. It has been shown to have the attributes of a serine protease capable of directly cleaving prothrombin to thrombin therefore leading to fibrin deposition. Previous work demonstrates $\mathrm{mfgl} / \mathrm{hfgl} 2$ expressed in the liver plays a pivotal role in the pathogenesis of experimental and human hepatic failure $(\mathrm{FHF})^{[11-18]}$. The nucleocapsid protein of mouse hepatitis virus 3 (MHV-3) induced transcription of $\mathrm{mfgl} 2$ and host hepatic nuclear factor $4 \alpha$ as a key transcription factor participated in the regulation of $\mathrm{mfg} 12$ gene expression $^{[14,23]}$. Interestingly, both MHV-3 (inducing the mfgl2 expression in Balb/cJ mouse) and SARS-CoV (the pathogen of SARS) belong to coronavirus family. The nucleocapsid proteins of both viruses identified can induce the hfgl $/ \mathrm{mfg} 12$ expression.

The SARS-CoV nucleocapsid $(\mathrm{N})$ protein is a 46 $\mathrm{kD}$ structural protein, and was demonstrated to activate other host genes. Besides its nucleocapsid assembly during the viral life cycle, the $\mathrm{N}$ protein has also been reported to activate the activator protein 1 (AP1) signal transduction pathway and induce apoptosis in COS-1 cells in the absence of growth factors ${ }^{[24,25]}$. Other results showed that the SARS-CoV N protein can significantly activate NF- $\kappa \mathrm{B}$ only in Vero E6 cells, which are susceptible to SARS-CoV infection, but not in Vero or HeLa cells $^{[7]}$. Yan et $a l^{[8]}$ investigated the roles of SARS-CoV proteins in regulation of the proinflammatory factor, and cyclooxygenase-2 (COX-2). SARS-CoV N protein was demonstrated to regulate the COX-2 gene expression. Electrophoretic mobility shift assay (EMSA) and chromatin immunoprecipitation (ChIP) demonstrated that SARS-CoV $\mathrm{N}$ protein bound directly to a NF-kappaB binding site and a CCAAT/enhancer binding protein $(\mathrm{C} / \mathrm{EBP})$ binding site. Protein mutation analysis revealed that a Lys-rich motif of $\mathrm{N}$ protein acted as a nuclear localization signal, and was essential for the activation of COX-2. Surjit et $a l^{[10]}$ showed that a short serine-rich stretch, a putative bipartite nuclear localization signal and self association through a C-terminal 209 amino acid interaction domain characterize this protein. These studies suggested that SARS-CoV N protein could enter the nuclear and affect host genes. Our results confirmed that $\mathrm{N}$ protein of SARS-CoV activated the hfgl 2 mRNA and protein expression, and mapping of $\mathrm{hfg} / 2$ promoter assay found the important regulation region, -817 to -417 (relative to transcription start site) in response to $\mathrm{N}$ protein. Further researches are undergoing in our laboratory to study the molecular interaction between virus proteins and the hfgl2 promoter.

Mapping of the promoter of hfgl2 gene determined the important regulatory region in the promoter from -817 to -417 (relative to transcription start site), and bioinformatics software provided the some positive cis-acting regulatory transcription factors binding sites. At present, we have not determined the precise cis-element(s) that are necessary for transcription. However, we have found some putative cis-elements such as HSTF, LEF and cEts and so on in the regulatory region. Studies now ongoing will firmly define relevant elements necessary for hfgl2 transcription.

Although data showed that other proteins of SARS-CoV besides $\mathrm{N}$ protein were involved in the gene expression regulation and mechanism of apoptosis, our study showed that neither $M$ nor S2 protein of SARS-CoV had effects on the expression of hfgl2 prothrombinase. It was recently shown that the 7a protein of SARS-CoV induced biochemical changes associated with apoptosis. Data indicate that the induction of apoptosis by the 7 a protein may be related to its ability to inhibit cellular translation and activate p38 MAPK $^{[25]}$. SARS-CoV $3 \mathrm{a}$ and $7 \mathrm{a}$ proteins-induced apoptosis in mammalian cells was confirmed, and membrane protein was currently identified to accelerate the induction of apoptosis in insect cells ${ }^{[26]}$.

In conclusion, three viral structural proteins, $\mathrm{M}, \mathrm{N}$ and S2 protein of SARS-CoV were expressed successfully in vitro using the eukaryotic expression constructions. Through real-time fluorescence quantitative RT-PCR and Western blot, we demonstrated up-regulated hfgl2 gene expression in response to $\mathrm{N}$ protein but not to $\mathrm{M}$ and $\mathrm{S} 2$ proteins. Luciferase assay suggested the $\mathrm{N}$ protein can activate the transcription of hfgl2 gene. Mapping of the promoter of hfgl2 gene determined the important regulatory region in the promoter from -817 to -417 (relative to transcription start site) and some important putative cis-elements, such as HSTF, LEF-1, c-ETS-2 and so on. This indicates that the SARS-CoV N protein may be involved in the pathogenesis of SARS, and this finding can be used in the development of therapeutics of SARS.

\section{Acknowledgements}

The authors thank Dr Gery Levy for his kindly providing plasmid pm166 and Professor Zhihong $\mathrm{Hu}$ for her kindly providing anti-SARS-CoV-M, anti-SARS-CoV-N and anti-SARSCoV-S2 polyclonal antibodies.

\section{REFERENCES}

1 Drosten C, Gunther S, Preiser W, et al. Identification of a novel coronavirus in patients with severe acute respiratory syndrome. N Engl J Med, 2003,348:1967-1976

2 Marra MA, Jones SJ, Astell CR, et al. The Genome sequence of the SARS-associated coronavirus. Science, 2003,300:1399-1404

3 Rota PA, Oberste MS, Monroe SS, et al. Characterization of a novel coronavirus associated with severe acute respiratory syndrome. Science, 2003,300:1394-1399 
4 He Y, Zhou Y, Wu H, et al. Mapping of antigenic sites on the nucleocapsid protein of the severe acute respiratory syndrome coronavirus. J Clin Microbiol, 2004,42: 5309-5314

$5 \mathrm{Hu} \mathrm{Y}$, Wen J, Tang L, et al. The M Protein of SARS-CoV: Basic structural and immunological properties. Genom Proteom Bioinform, 2003,1:118-130

6 Chen $\mathrm{H}$, Wurm $\mathrm{T}$, Britton $\mathrm{P}$, et al. Interaction of the coronavirus nucleoprotein with nucleolar antigens and the host cell. J Viro, 2002,76:5233-5250

7 Liao QJ, Ye LB, Timani KA, et al. Activation of NF-kappaB by the full-length nucleocapsid protein of the SARS coronavirus. Acta Biochim Biophys Sin (Chinese), 2005,37:607-612

8 Yan X, Hao Q, Mu Y, et al. Nucleocapsid protein of SARS-CoV activates the expression of cyclooxygenase-2 by binding directly to regulatory elements for nuclear factor-kappa B and CCAAT/enhancer binding protein. Int J Biochem Cell Biol, 2006,38:1417-1428

9 Law HK, Cheung CY, Ng HY, et al. Chemokine up-regulation in SARS-coronavirus-infected, monocyte-derived human dendritic cells. Blood, 2005,106: 2366-2374

10 Surjit M, Liu B, Jameel S, et al. The SARS coronavirus nucleocapsid protein induces actin reorganization and apoptosis in COS-1 cells in the absence of growth factors. Biochem J, 2004,383 (Pt 1):13-18

11 Zhu CL, Yan WM, Zhu F, et al. Fibrinogen-like protein 2 fibroleukin expression and its correlation with disease progression in murine hepatitis virus type 3-induced fulminant hepatitis and in patients with severe viral hepatitis B. World J Gastroenterol, 2005,11:6936-6940

12 Ding JW, Ning Q, Liu MF, et al. Expression of the fgl2 and its protein product (prothrombinase) in tissues during murine hepatitis virus strain-3 (MHV-3) infection. Adv Exp Med Biol, 1998,440:609-618

13 Levy GA, Liu M, Ding J, et al. Molecular and functional analysis of the human prothrombinase gene (HFGL2) and its role in viral hepatitis. Am J Pathol, 2000,156: 1217-1225

14 Ning Q, Liu M, Kongkham P, et al. The nucleocapsid protein of murine hepatitis virus type 3 induces transcription of the novel fgl2 prothrombinase gene. J Biol Chem, 1999,274:9930-9936

15 Marazzi S, Blum S, Hartmann R, et al. Characterization of human fibroleukin, a fibrinogen-like protein secreted by T lymphocytes. J Immunol, 1998,161:138-147

16 Marsden PA, Ning Q, Fung LS, et al. The Fgl2/fibroleukin prothrombinase contributes to immunologically mediated thrombosis in experimental and human viral hepatitis. J Clin Invest, 2003,112:58-66

17 Ning Q, Sun Y, Han M, et al. Role of fibrinogen-like protein 2 prothrombinase/fibroleukin in experimental and human allograft rejection. J Immunol, 2005,174:74037411

18 Robertson M. Fgl2: link between hepatitis B and SARS. Drug Discov Today, 2003,8:768-770

19 Yan WM, Ning Q, Luo XP. Liver impairment in MHV-3 induced murine SARS model. Chin J Infect Dis (Chinese), 2006,24:361-365

20 Ma SW, Wang ZH, Zhang Ke, et al. Expression of recombinant SARS associated coronavirus structural protein in Escherchiacoli BL21. Med J Chin PLA (Chinese), 2004,29:748-751

21 Pei F, Zheng J, Gao ZF, et al. Lung pathology and pathogenesis of severe acute respiratory syndrome: a report of six full autopsies. Chin J Pathol (Chinese), 2005,34: 656660

22 Yan WM, Zhu CL, Luo XP, et al. Establishment of a substitutive SARS murine model and its application in SARS study. J Clin Immunol (Chinese), 2005,Supplement(1):166-167

23 Ning Q, Lakatoo S, Liu M, et al. Induction of prothrombinase fgl2 by the nucleocapsid protein of virulent mouse hepatitis virus is dependent on host hepatic nuclear factor-4 alpha. J Biol Chem, 2003,278:15 541-15 549

24 He R, Leeson A, Andonov A, et al. Activation of AP-1 signal transduction pathway by SARS coronavirus nucleocapsid protein. Biochem Biophys Res Commun, 2003,311:870-876

25 Opecky-Bromberg SA, Martinez-Sobrido L, Palese P, et al. 7a protein of severe acute respiratory syndrome coronavirus inhibits cellular protein synthesis and activates p38 mitogen-activated protein kinase. J Virol, 2006,80: 785-793

26 Ai CW, Chan ZR, Yang DG, et al. Accelerated induction of apoptosis in insect cells by baculovirus-expressed SARS-CoV membrane protein. FEBS Lett, 2006,580: 3829-3834

(Received March 11, 2009) 